\title{
The Effects of Lactobacillus rhamnosus GG on Mechanical Properties of Long Bones and Body Composition in Immunodeficient Mice
}

\author{
Violetta Naughton'1, Dorian Dixon², Lisa Rainey³, Cormac McGarrigle², \\ Patrick J. Naughton ${ }^{*}$ \\ ${ }^{1}$ Biomedical Sciences Research Institute, School of Biomedical Sciences, Ulster University, Coleraine, UK, \\ ${ }^{2}$ Engineering Research Institute, School of Engineering, Ulster University, Jordanstown, UK \\ ${ }^{3}$ Centre for Cancer Research and Cell Biology, School of Medicine, Dentistry and Biomedical Sciences, Queen's \\ University, Belfast, UK \\ Email: *pj.naughton@ulster.ac.uk
}

Received 29 October 2015; accepted 18 March 2016; published 21 March 2016

Copyright (C) 2016 by authors and Scientific Research Publishing Inc.

This work is licensed under the Creative Commons Attribution International License (CC BY). http://creativecommons.org/licenses/by/4.0/

(c) (i) Open Access

\section{Abstract}

This study aimed to assess whether a daily supplementation with Lactobacillus rhamnosus GG (LGG) can alter the body composition as well as bone turnover and the mechanical properties of the bones in systemic immunodeficiency. For this, we use an in vivo model of severe combined immunodeficient (scid) mice supplemented daily with LGG over 8 weeks period. After 8 weeks of the treatment, the animals were assessed by DEXA and then the blood was collected from the hepatic portal vein for bone turnover biomarkers; post-mortem, the caecum was removed for isolation and enumeration of the gut bacteria, while femurs/tibiae were excised for the assessment of their mechanical properties. LGG treatment affected the composition of the gut microbiota at the order level, and it decreased $(P=0.002)$ total adipose tissue content. Moreover, LGG treatment decreased the stiffness $(\mathrm{N} / \mathrm{mm})$ of both femurs $(P=0.008)$ and tibiae $(P=0.011)$. To conclude, this study shows that dietary supplementation with LGG in immunodeficient animals can alter mechanical properties of the long bones and concomitantly it can modify host's total adiposity level.

\section{Keywords}

Lactobacilli, Bone, Body Composition

\footnotetext{
*Corresponding author.
} 


\section{Introduction}

The role of gut microbiota in the regulation of host metabolism including mineral and lipid metabolism has been previously reported from in vivo models as well as from human trials. More recently, it has been shown that germ-free (GF) animals display greater bone mass with reduced bone resorption when compared to conventional animals with an unaltered gut microbiome. In addition, the reconstitution of the gut microbiome in GF animals resulted in bone mass reduction with outcomes comparable to their conventional counterparts [1]. On the other hand, it has been shown in humanised GM animals that modification of the gut microbiota even with a single bacterial strain e.g. Lactobacillus rhamnosus can attenuate the hosts lipid and amino acid metabolism [2]. Previous work on the effects of gut microbiota modification on a host's mineral and lipid metabolism was obtained from immunocompetent animals [1]-[3] and to our knowledge there was no information available on the effects of the gut microbiota on the bone and/or lipid metabolism in systemic immunodeficiency. Therefore, the aim of this study was to assess whether modifying the gut microflora with Lactobacillus rhamnosus GG (LGG) could alter bone turnover, the mechanical properties of the developing bones and body composition in systemic immunodeficiency in vivo. For this, we use severe combined immunodeficient (scid) mice [4] and LGG that originates from infant faeces [5] and has been shown to affect the host's glucose, lipid and mineral metabolism in a conventional, immunocompetent murine in vivo models [3] [6].

\section{Materials and Methods}

\subsection{Animals and Experimental Protocol}

Female scid mice obtained from an in-house colony $(n=18,7.5$-weeks old) were weighted and allocated into two groups ( $\mathrm{n}=9$ per group) according to their body mass (BM) i.e.: $16.02 \pm 1.66 \mathrm{~g}$ (mean \pm CI 95\%) for the control group and $16.84 \pm 1.63 \mathrm{~g}$ (mean \pm CI 95\%) for the treatment group assuring homogeneity of variance between groups (4.6 and 4.5 for the control and treatment group, respectively). Over a period of eight consecutive weeks, the animals allocated to the treatment group (referred in the text as the LGG group) were fed daily with Lactobacillus rhamnosus GG (LGG) at $8.8 \times 10^{9} \mathrm{CFU} / 100 \mathrm{~g} \mathrm{BM} / 24 \mathrm{~h}$ on the background of irradiated standard diet for growing mice (2918 Teklad Global 18\% protein rodent diet, Harlan, Madison, USA). The animals allocated to the control group were fed with the same diet (2918 Teklad Global 18\% protein rodent diet, Harlan, Madison, USA) but were not supplemented with LGG. The daily dose of LGG supplied to the animals allocated to the LGG group was based on the previous results by Gill and Rutherfurd [7] evaluated in BALB/c mice and confirmed as effective at increasing the LGG counts in the caecum of scid mice in a pilot test carried out in-house prior to the commencement of the experiment. All animals were housed in a temperature-controlled environment $\left(22^{\circ} \mathrm{C} \pm 2^{\circ} \mathrm{C}\right)$ with a $12: 12 \mathrm{~h}$ light-dark cycle, in individually ventilated cages supplied with sterile bedding and nesting material, and provided ad libitum with sterile water and feed (2918 Teklad Global $18 \%$ protein rodent diet, Harlan, Madison, USA) containing 18.6\% crude protein, 6.2\% fat (ether extract), $44.2 \%$ available carbohydrates (total carbohydrates-neutral detergent fibre), 3.5\% crude fiber, 14.7\% neutral detergent fiber, $5.35 \%$ ash and calculated estimate of metabolizable energy of $13.0 \mathrm{~kJ} / \mathrm{g}$. The 2918 diet is commercially available as fix formula that is based on the following ingredients: ground wheat, ground corn, wheat middlings, dehulled soybean meal, corn gluten meal, soybean oil, vitamin and mineral mix including $1 \%$ of calcium and $0.7 \%$ of phosphorus, as declared by the manufacturer (Harlan, Madison, USA). All ethical and legal permissions/licences were obtained prior to the start of the experiments in accordance to Animals (Scientific Procedures) Act 1986. After 8 weeks of the treatment, mice were anesthetized with an intraperitoneal injection of $3 \mu \mathrm{l} / 1 \mathrm{~g} \mathrm{LBM}$ of Hypnorm (Chemos GmbH, Regenstauf, Germany) and Hypnovel (midazolam; Roche Diagnostics Ltd, Burgess Hill, UK) in water in a ratio of 1:1:2 Hypnorm/Hypnovel/water. The body composition measurements were carried out on anesthetized animals using dual-energy x-ray absorptiometry (DEXA; the Lunar PIXImus Densitometer, GE Medical Systems, Fitchburg, USA) to assess total bone mineral content ( $t$ BMC, g), total bone mineral density ( $t \mathrm{BMD}, \mathrm{g} / \mathrm{cm}^{2}$ ), and total soft (g), lean $(\mathrm{g})$ and adipose (g) tissue content. Thereafter, the blood was collected from the hepatic portal vein and after processing, the aliquoted samples of plasma and serum were frozen at $-80^{\circ} \mathrm{C}$ for subsequent analyses. At the completion of blood withdrawal, the animals were killed by the cervical dislocation. At death, the caecum was removed for isolation and enumeration of bacteria, while left/ right femurs and tibiae were excised, cleaned of all muscle and connective tissues, wrapped in cotton gauze soaked in $0.90 \% \mathrm{w} / \mathrm{v}$ of $\mathrm{NaCl}$, placed in an air tight plastic bags and then stored at $-20^{\circ} \mathrm{C}$ for subsequent analyses. 


\subsection{Analyses, Measurements and Calculations}

Fresh caecum samples were weighed and homogenized on ice with a Janke-Kunkel Ultra-Turrax T25 homogenizer (Ascher, The Netherlands) at 20,000 rpm for 20 seconds in maximum recovery diluent (MRD) (CM0733; Oxoid), to provide a 100-g liter ${ }^{-1}$ suspension [8]. Subsequently, each sample was serially diluted to $10^{-8}$ with the same diluent (MRD). Dilutions of the samples were inoculated in duplicate onto selective and nonselective agars: De Man, Rogosa, Sharpe (MRS) agar, (CM0359, Oxoid) with nalidixic acid (NAL) for enumeration of LGG [9]; MRS agar for enumeration of total lactobacilli, Columbia agar (CM331, Oxoid) containing 5\% (vol/vol) defibrinated horse blood for total anaerobic bacteria and MacConkey agar no. 3 (CM0115, Oxoid) for enumeration of enterobacteria. Inoculated anaerobe-selective agar plates were incubated in an anaerobic cabinet (Don Whitley Scientific, Shipley, United Kingdom) at $37^{\circ} \mathrm{C}$ for $48 \mathrm{~h}$ (Columbia agar plates) and $72 \mathrm{~h}$ (MRS, MRS with NAL plates), while MacConkey agar plates were incubated aerobically at $37^{\circ} \mathrm{C}$ for $18 \mathrm{~h}$. All agar plates were examined for bacteria colony forming units (CFU); the average of the viable counts (accounting for dilution factor used) were $\log _{10}$-transformed and normalised per $1 \mathrm{~g}$ of wet weight i.e. the weight of fresh caecum samples (tissue plus content) taken prior to the homogenisation.

The femurs were thawed at room temperature, placed in a sterile petri dish filled with sterile $0.90 \% \mathrm{w} / \mathrm{v}$ of $\mathrm{NaCl}$ and scanned with Lunar PIXImus (GE Medical Systems) for femoral BMC (fBMC, g) and femoral BMD $\left(f B M D, g / \mathrm{cm}^{2}\right)$. The mechanical properties of the long bones i.e. stiffness $(\mathrm{N} / \mathrm{mm})$, ultimate breaking load $(\mathrm{N})$ and deflection at break $(\mathrm{mm})$ were evaluated using an Instron 3345 tensile tester with a loading rate of $0.2 \mathrm{~mm} / \mathrm{s}$ and the distance between the supports of $8 \mathrm{~mm}$ [10].

Serum osteocalcin was determined by radioimmunoassay with reagents of DRG Instruments (Osteocalcin (Mouse) ELISA, DRG Instruments, Marburg, Germany); the intra- and interassay coefficient of variations were $3.34 \%$ and $4.00 \%$, respectively. Serum osteoclast-derived tartrate-resistant acid phosphatase form $5 \mathrm{~b}$ (TRAP 5b) was determined by Solid phase immunofixed enzyme activity assay with reagents of Immunodiagnostic Systems Ltd (MouseTRAP ${ }^{\text {TM }}$ Assay, Immunodiagnostic Systems Ltd, Boldon, Tyne \& Wear, UK); the intra- and interassay coefficient of variations were $3.31 \%$ and $1.78 \%$, respectively. Plasma $\mathrm{N}$-terminal propeptide of type I procollagen (PINP) was determined by radioimmunoassay with reagents of Immunodiagnostic Systems Ltd. (Rat/Mouse PINP EIA, Immunodiagnostic Systems Ltd., Boldon, Tyne \& Wear, UK); the intra- and inter-assay coefficient of variations were $1.89 \%$ and $4.40 \%$, respectively.

All obtained data sets were checked for the normality of the distribution using Kolmogorov-Smirnov (K-S test) test of goodness-of-fit. The K-S test outcomes provided no evidence ( $P>0.05$ for all data sets) against the null hypothesis that each sample has been drawn from a normal distribution. Therefore the effect of the LGG treatment was evaluated with a one-way MANOVA with a group (LGG vs. control) as independent variable and the following sets of dependent variables: 1) bacteria counts in the caecum, 2) body weight and weight gain, 3) body composition parameters, 4) total bone mineral density and contents, 5) long bone mineral composition, 6) parameters of mechanical properties of femurs, 7) parameters of mechanical properties of tibiae, 8) bone metabolism biomarkers. Within each data set the homogeneity of variances and covariances was tested with SPSS Levene's test and Box's M test respectively with the latter showing the outcomes $P>0.001$ for all matrixes tested. The effect of the treatment on feed utilisation was assessed with a one-way ANOVA with a group (LGG vs. control) as independent variable and feed utilisation (g) normalised for $100 \mathrm{~g}$ live body mass and measured over the entire experimental period of 8-weeks. In all analyses, $P<0.05$ was assigned as the level of statistical significance. The statistical analyses were performed with SPSS (IBM ${ }^{\circledR}$, SPSS Statistics, v. 20.0.0).

\section{Results}

A one-way MANOVA showed a significant main effect for treatment, (Wilks' $\lambda=0.471$, exact $P=0.046$, power to detect the effect was 0.673 ) on bacteria counts in caecum. The main univariate effects were significant for LGG counts (exact $P=0.021$ ), total lactobacilli counts (exact $P=0.025$ ) and total anaerobes counts (exact $P=$ 0.025 ) but not for the enterobacteria counts (exact $P=0.837$ ); see Figure 1 for details.

A one-way MANOVA showed no main effect of treatment (Wilks' $\lambda=0.940$, exact $P=0.114$ ) for body weight gain over the entire experiment (3.86 v vs. $4.36 \mathrm{~g}$ for LGG and control group, respectively) and for the body mass of animals recorded at the end of the experiment (20.70 vs. $20.38 \mathrm{~g}$ for LGG and control group, respectively). There was no effect for treatment detected (exact $P=0.97$ ) for feed utilisation (65.2 vs. $65.4 \mathrm{~g} / 100 \mathrm{~g}$ live body mass, for LGG and control group, respectively) measured over the entire experimental period of 


\section{8-weeks.}

However, the main multivariate effect for treatment on the total soft tissue, lean and adipose tissue contents was significant (Wilks' $\lambda=0.406$, exact $P=0.007$, power to detect the effect was 0.896 ). Significant univariate main effects for treatment were detected for adipose tissue content (exact $P=0.002$ ), but not for total soft tissue (exact $P=0.433$ ) and lean tissue content (exact $P=0.691$ ); see Figure 2 for details.

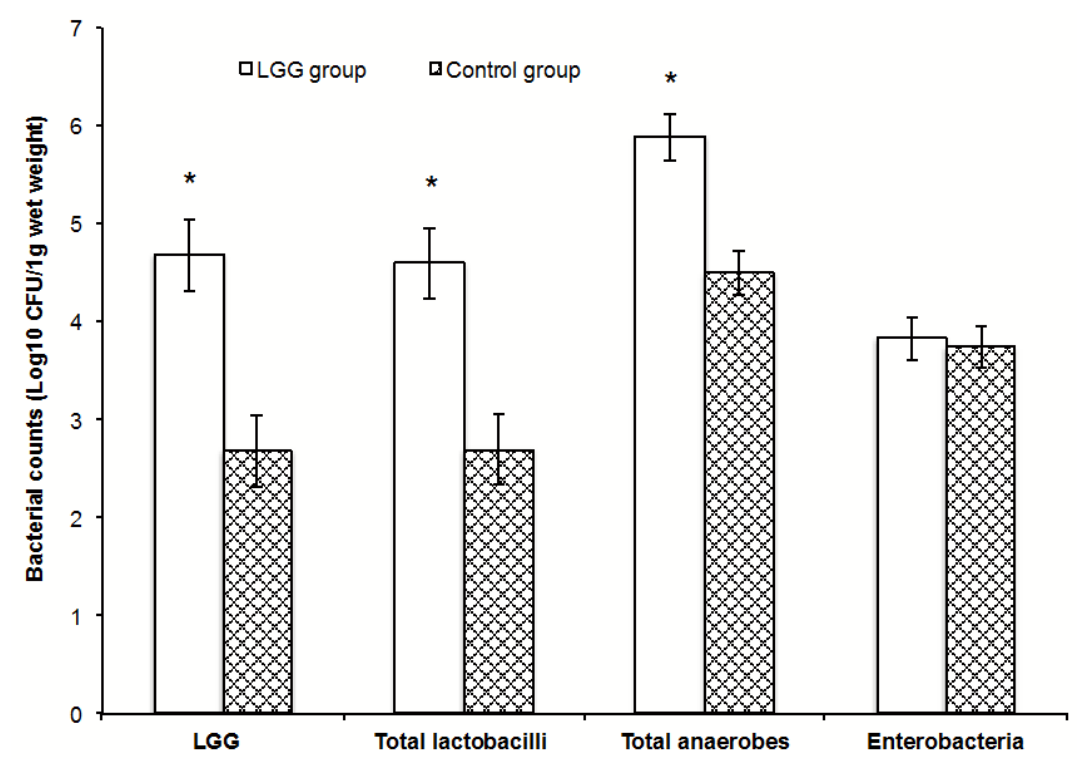

Figure 1. Bacterial counts from the caecal tissue and content of growing scid mice supplemented daily with Lactobacillus rhamnosus GG (LGG group) over a period of 8 consecutive weeks. Total heights of open and filled bars represent mean values \pm CI $95 \%$. Values marked with an asterisk are significantly different from the corresponding control group fed standard diet only $\left({ }^{*} P<0.05\right)$; total $N=18$; $\mathrm{n}=9$ per group.

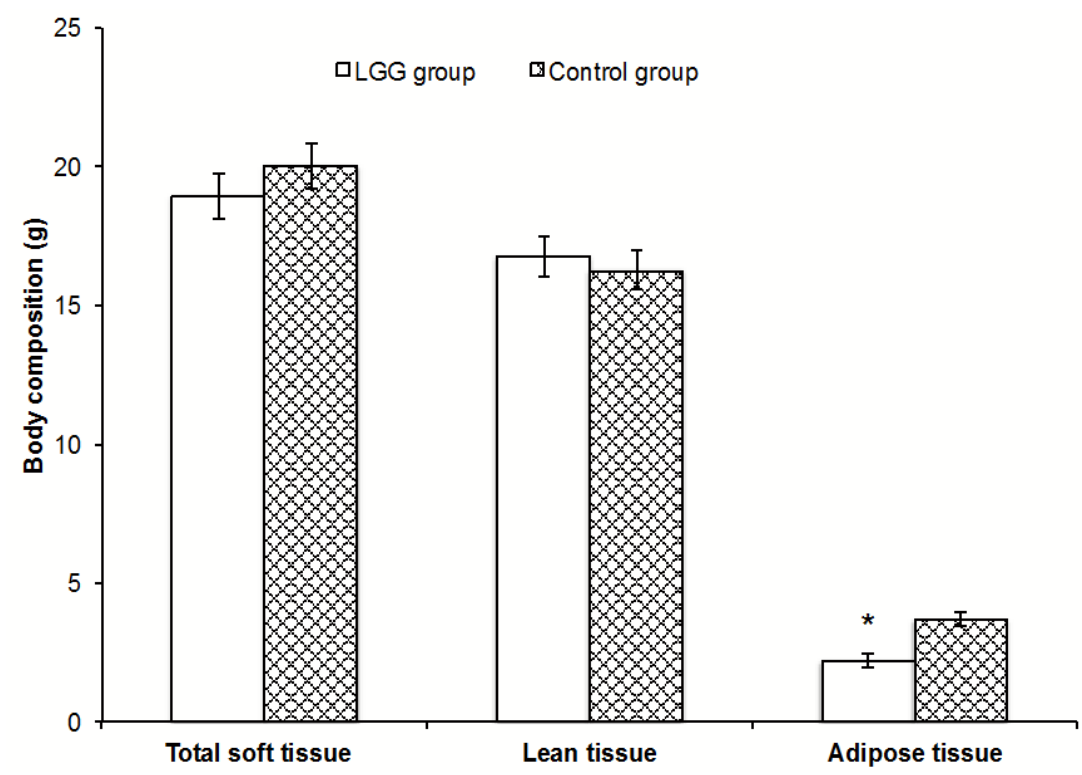

Figure 2. Body composition of growing scid mice supplemented daily with Lactobacillus rhamnosus GG (LGG group) over a period of 8 consecutive weeks. Total heights of open and filled bars represent mean values \pm CI 95\%. Values marked with an asterisk are significantly different from the corresponding control group fed standard diet only ( $\left.{ }^{*} P<0.01\right)$; total $N=18 ; \mathrm{n}=9$ per group. 
A one-way MANOVA showed no main effect for treatment (Wilks' $\lambda=0.972$, exact $P=0.819$ ) on $t$ BMC (0.46 g, mean value for all animals) and $t$ BMD $\left(0.05 \mathrm{~g} / \mathrm{cm}^{2}\right.$, mean value for all animals). Similarly, there was no main multivariate effect for treatment detected (Wilks' $\lambda=0.812$, exact $P=0.096$ ) for femurs $f$ BMC $(0.03$ g, mean value for all animals) and fBMD $\left(0.07 \mathrm{~g} / \mathrm{cm}^{2}\right.$, mean value for all animals).

However, the main multivariate effect for treatment on the mechanical properties of the femurs showed tendency towards significance (Wilks' $\lambda=0.773$, exact $P=0.055$, power to detect the effect was 0.619 ) and thus the univariate main effects were examined. Significant univariate main effect for treatment for the stiffness $(\mathrm{N} / \mathrm{mm}$ ) was determined to be significant (39.58 vs. 49.86 for LGG and control group, respectively, exact $P=$ 0.008), however not for ultimate breaking load (exact $P=0.140 ; 21.21 \mathrm{~N}$, mean value for all animals) and deflection at break (exact $P=0.209 ; 0.58 \mathrm{~mm}$, mean value for all animals). Furthermore, a one-way MANOVA showed significant main effect for treatment on the mechanical properties of the tibiae (Wilks' $\lambda=0.740$, exact $P=0.041$, power to detect the effect was 0.666). Similarly, as in the case of femurs, significant univariate main effect for treatment on the stiffness (N/mm) was determined (18.97 vs. 30.01 for LGG and control group, respectively, exact $P=0.011$ ), however not for ultimate breaking load (exact $P=0.128 ; 12.51 \mathrm{~N}$, mean value for all animals) and defection at break (exact $P=0.506 ; 0.86 \mathrm{~mm}$, mean value for all animals).

Tested bone metabolism biomarkers i.e. serum osteocalcin, serum TRAP 5b and plasma PINP were not affected by the treatment i.e. (Wilks' $\lambda=0.854$, exact $P=0.683$ ).

\section{Discussion}

The results of this study showed that the treatment with Lactobacillus rhamnosus GG (LGG) on the background of nutritionally balanced and adequate for age/body weight diet affects the body composition and the mechanical properties of the long bones in immunodeficient animals. The animals treated with LGG in this study showed a reduced stiffness of the long bones (by $\sim 20 \%$ and $\sim 37 \%$ of femurs and tibiae, respectively) when compared to the control mice. The stiffness of bones is primarily imparted by mineral density however we found no effects of LGG on the mineral density and content of the whole skeleton or on the weight-bearing segments of the skeleton. Also, bone formation biomarkers (serum osteocalcin (OC) and plasma N-terminal propeptide of type I procollagen (PINP), and bone resorption biomarker (serum tartrate-resistant acid phosphatase (TRAP, band 5b), from LGG-treated animals were no different to the respective values obtained from the control mice. Thus, the reduced stiffness of the long bones, without other observable mechanical or mineral effects in LGG-treated mice could perhaps indicate a modification of the structural properties of the bone organic matrix, not assessed in this study. It has been previously shown that changes to the structure of the organic matrix without significant changes to the bone mineral properties or total collagen accumulation results in attenuated structure of the bone [11]. Characterisation of the possible effects of gut microbiome modification on the properties of the bone organic matrix would however require further investigation.

This study showed that a change in the gut microflora also changes the body composition of immunodeficient animals. The absolute values of the body composition parameters showed the significant effects of LGG on the total adipose tissue content i.e., LGG led to an $~ 18 \%$ drop in adipose tissue content. Moreover, supplementation with LGG changed the relative ratio between lean and adipose tissue i.e. 8 lean to 1 adipose tissue in LGG treated vs. 4 lean to 1 adipose tissue in the control animals. Kim et al. [3] in a study on immunocompetent (C57BL) mice observed that oral supplementation with LGG decreased the end body weight and also decreased mesenteric and liver fat but only in animals fed high fat diet and not in mice fed standard diet. The authors suggested that anti-adipose effects of LGG may only be observed when animals received higher than normally required amount of dietary energy. However, their study did not evaluate if, or to what extend high fat diet affected the microbiome composition of the gut. Several studies have shown that a high content of dietary fat in itself significantly changes the gut microbiome composition [12] possibly via increased bile acids secretion [13]. Moreover, the results of previous studies in immunocompetent animal models [14] and in human subjects [15] have shown the ability of the gut microbiome to affect the host's metabolic phenotype, including adiposity levels. Martin et al. [2] in a humanised microbiome mouse model showed that supplementation of immunocompetent animals with L. rhamnosus decreased the populations of bacterial strains associated with high levels of both tauro-conjugated and unconjugated bile acids in the gut lumen; moreover, the hepatic acetate: propionate ratio was reduced of which the latter have been previously shown to lower serum lipids [16]. Thus, it could be suggested that the changes to the gut microbiota observed in the animals treated with LGG in this study, affected 
adipose tissue depositing via modification of the host's lipid metabolism. However, this would require further investigation on gut microbiome and metabolism of lipids in systemic immunodeficiency.

In this study, we used an in vivo model of growing scid mice in which immunodeficiency mutation affected the development of both $\mathrm{T}$ and $\mathrm{B}$ cells, while natural killer (NK) cells, macrophage and granulocytes numbers were significantly reduced. To our knowledge, there are no data available on the effects on this type of immunodeficiency on the body composition or the bone, while the results of previous studies on comparison of bone phenotypes in athymic vs. euthymic mice are not uniform. McCauley et al. [17] demonstrated that growing athymic, T-cell deficient (nude) mice have lower body weight, shorter tibia, reduced ash content of dry bone and lower serum calcium level when compared to euthymic mice. Cenci et al. [18] on the other hand, have shown that growing nude mice present normal phenotypes including bone mineral densities and bone morphology when compared to immunocompetent littermates. In this study, we did not have an opportunity to assess immunocompetent animals, thus we cannot directly contrast the LGG effects on bone and body composition observed in our scid mice to immunocompetent animals. Therefore, it can be only speculated that specific gut microbiota e.g. the strains with particular metabolic capabilities are able to affect an immunocompromised host with effects e.g. on the bone that are relevant to health. Furthermore, in this study we used one sex of growing animals. The differences in the rate of growth between male and females scid mice are acknowledged and the use of one sex of animals allowed us to assure homogeneity of between and within group variance. However, this approach does not allow for an extrapolation of our findings to the other sex due to possible confounding endocrine effects on the bone and adiposity level. A larger animal trial with sex, age and immune status as experimental variables is required to confirm our observations.

\section{Conclusion}

To conclude, this study shows that the introduction of LGG into immunodeficient female mice can alter the mechanical properties of the long bones and modify total adiposity level during growth. The results of this study merit further investigation into the mechanisms underlying these changes, which might allow translation of the research findings into clinical practice.

\section{Conflicts of Interest}

The authors declare that there is no conflict of interest regarding the publication of this paper.

\section{References}

[1] Sjögren, K., Engdahl, C., Henning, P., Lerner, U.H., Tremaroli, V., Lagerquist, M.K., Bäckhed, F. and Ohlsson, C. (2012) The Gut Microbiota Regulates Bone Mass in Mice. Journal of Bone and Mineral Research, 27, 1357-1367. http://dx.doi.org/10.1002/jbmr.1588

[2] Martin, F.-P.J., Wang, Y., Sprenger, N., Yap, I.K.S., Lundstedt, T., Lek, P., Rezzi, S., Ramadan, Z., van Bladeren, P., Fay, L.B., Kochhar, S., Lindon, J.C., Holmes, E. and Nicholson, J.K. (2008) Probiotic Modulation of Symbiotic Gut Microbial-Host Metabolic Interactions on a Humanized Microbiome Mouse Model. Molecular Systems Biology, 4, 157. http://dx.doi.org/10.1038/msb4100190

[3] Kim, S.W., Park, K.-Y., Kim, B., Kim, E. and Hyun, C.-K. (2013) Lactobacillus rhamnosus GG Improves Insulin Sensitivity and Reduces Adiposity in High-Fat Diet-Fed Mice through Enhancement of Adiponectin Production. Biochemical and Biophysical Research Communications, 431, 258-263.

[4] Bosma, M.J. and Caroll, A.M. (1991) The Scid Mouse Mutant-Definition, Characterisation, and Potential Uses. Annual Review of Immunology, 9, 323-350. http://dx.doi.org/10.1146/annurev.iy.09.040191.001543

[5] Heilig, H.G.H.J., Zoetendal, E.G., Vaughan, E.E., Marteau, P., Akkermans, A.D.L. and de Vos, W.M. (2002) Molecular Diversity of Lactobacillus spp. and Other Lactic Acid Bacteria in The Human Intestine as Determined by Specific Amplification of 16S Ribosomal DNA. Applied and Environmental Microbiology, 68, 114-123. http://dx.doi.org/10.1128/AEM.68.1.114-123.2002

[6] Naughton, V., McSorely, E. and Naughton, P.J. (2011) Changes in Calcium Status in Aged Rats Fed Lactobacillus rhamnosus GG and Bifidobacterium lactis and Oligofructose-Enriched Inulin. Applied Physiology, Nutrition, and Metabolism, 36, 161-165. http://dx.doi.org/10.1139/H10-088

[7] Gill, H.S. and Rutherfurd, K.J. (2001) Viability and Dose-Response Studies on the Effects of the Immunoenhancing Lactic Acid Bacterium Lactobacillus rhamnosus in Mice. British Journal of Nutrition, 86, 285-289. 
[8] Naughton, P.J., Mikkelsen, L.L. and Jensen, B.B. (2001) Effects of Nondigestible Oligosaccharides on Salmonella enterica Serovar Typhimurium and Non-Pathogenic Escherichia coli in the Pig Small Intestine in Vitro. Applied and Environmental Microbiology, 67, 3391-3395. http://dx.doi.org/10.1128/AEM.67.8.3391-3395.2001

[9] DebMandal, M., Mandal, S. and Pal, N.K. (2012) Detection of Intestinal Colonization of Probiotic Lactobacillus rhamnosus by Stool Culture in Modified Selective Media. Asian Pacific Journal of Tropical Disease, 2, 205-210. http://dx.doi.org/10.1016/S2222-1808(12)60047-4

[10] Schriefer, J.L., Robling, A.G., Warden, S.J., Fournier, A.J., Mason, J.J. and Turner, C.H. (2005) A Comparison of Mechanical Properties Derived from Multiple Skeletal Sites in Mice. Journal of Biomechanics, 38, 467-475.

[11] Boskey, A.L., Goldberg, M., Kulkarni, A. and Gomez, S. (2006) Infrared Imaging Microscopy of Bone: Illustrations from a Mouse Model of Fabry Disease. Biochimica et Biophysica Acta, 1758, 942-947. http://dx.doi.org/10.1016/j.bbamem.2006.02.019

[12] Janssen, A.W. and Kersten, S. (2015) The Role of the Gut Microbiota in Metabolic Health. FASEB Journal, 29, fj-14269514. http://dx.doi.org/10.1096/fj.14-269514

[13] Islam, K.B., Fukiya, S., Hagio, M., Fujii, N., Ishisuka, S., Ooka, T., Ogura, Y., Hayashi, T. and Yokota, A. (2011) Bile Acid Is a Host Factor That Regulates the Composition of the Cecal Microbiota in Rats. Gastroenterology, 141, 17731781. http://dx.doi.org/10.1053/j.gastro.2011.07.046

[14] Turnbaugh, P.J., Ley, R.E., Mahowald, M.A., Magrini, V., Mardis, E.R. and Gordon, J.I. (2006) An Obesity-Associated Gut Microbiome with Increased Capacity for Energy Harvest. Nature, 444, 1027-1031. http://dx.doi.org/10.1038/nature05414

[15] Vrieze, A., van Nood, E., Holleman, F., Salojärvi, J. Kootte, R.S., Bartelsman, J.F.W.M., Dallinga-Thie, G.M., Ackermans, M.T., Serlie, M.J., Oozer, R., Derrien, M., Druesne, A., van Hylckama Vlieg, J.E.T., Blocks, V.W., Groen, A.K., Heilig, H.G.H.J., Zoetendal, E.G., Stroes, E.S., de Vos, W.M., Hoekstra, B.L. and Nieuwdroop, M. (2012) Transfer of Intestinal Microbiota from Lean Donors Increases Insulin Sensitivity in Individuals with Metabolic Syndrome. Gastroenterology, 143, 913-916. http://dx.doi.org/10.1053/j.gastro.2012.06.031

[16] Wong, J.M., de Souza, R., Kendall, C.W., Emam, A. and Jenkins, D.J. (2006) Colonic Health: Fermentation and Short Chain Fatty Acids. Journal of Clinical Gastroenterology, 40, 235-243. http://dx.doi.org/10.1097/00004836-200603000-00015

[17] McCauley, L.K., Rosol, T.J., Capen, C.C. and Horton, J.E. (1989) A Comparison of Bone Turnover in Athymic (Nude) and Euthymic Mice: Biochemical, Histomorphometric, Bone Ash and in Vitro Studies. Bone, 10, 29-34. http://dx.doi.org/10.1016/8756-3282(89)90144-0

[18] Cenci, S, Weitzmann, M.N., Roggia, C., Namba, N., Novack, D., Woodring, J. and Pacifici, R. (2000) Estrogen Deficiency Induces Bone Loss by Enhancing T-Cell Production of TNF- $\alpha$. Journal of Clinical Investigations, 106, 12291237. http://dx.doi.org/10.1172/JCI11066 